\title{
La Saône en crue : dynamique d'un hydrosystème anthropisé
}

\author{
par Laurent Astrade, \\ UMR 5600 du CNRS, Lyon
}

\begin{abstract}
M. Laurent Astrade est lauréat du prix Henri Milon 1997 et est actuellement en stage post-doctoral au Québec (Centre d'Etudes Nordiques de l'Université de Laval)
\end{abstract}

\section{I 口 INTRODUCTION}

La Saône prend sa source dans les Vosges et se jette, après $480 \mathrm{~km}$, dans le Rhône à Lyon (figure 1). Son régime se distingue par un minimum marqué en août et une période de hautes eaux en hiver (module : $442 \mathrm{~m}^{3 /} / \mathrm{s}$ ) aux crues nombreuses et lentes dans leur évolution et leur propagation. Les crues sont à l'origine de la spécificité écologique du Val de Saône, à la fois élément essentiel de la qualité du milieu et forte entrave pour les sociétés riveraines. Si les études sur la Saône sont toutes guidées par la problématique de l'inondation, notre travail s'attache plus à analyser l'hydrosystème en période de crue, à plusieurs échelles spatiales et temporelles, et plus précisément à connaître sa dynamique sous l'influence de la géomorphologie de son bassin et de l'anthropisation de son cours, à évaluer les conséquences des forts débits sur l'évolution du cours d'eau et des berges, et à observer les effets des hautes eaux sur la ripisylve.

\section{GÉOMORPHOLOGIE DE LA SAÔNE ACTUELLE ET DYNAMIQUE DES CRUES}

Le fonctionnement de l'hydrosystème est d'abord affecté par des processus géomorphologiques globaux et des changements climatiques majeurs à l'échelle de 1000 à 10000 ans. Après avoir examiné le régime des crues de la Saône à l'aval du bassin depuis 1920, grâce aux débits journaliers, et énoncé le changement du régime des crues (augmentation de la fréquence depuis une quinzaine d'années et glissement des périodes de hautes eaux de l'automne au printemps), nous présentons la géologie du bassin et l'histoire de l'aménagement de la rivière. L'étude de la morphologie et de l'hydrologie du cours actuel de la Saône à l'échelle du bassin versant nous permet de montrer les implications de ces deux facteurs qui, en conditionnant le profil en long, les débits liquides et les débits solides, la sédimentation et l'érosion, le champ d'inondation..., sont les fondements de la dynamique de la Saône en crue.
La méthode consiste à quantifier longitudinalement de Corre ( $\mathrm{Pk} 407)$ à Lyon $(\mathrm{Pk} 0)$ plusieurs variables à l'aide de 400 profils en travers du lit mineur et du lit majeur de la Saône (CNR-Beture, 1989). Faiblesse de la pente globale $(0,15 \mathrm{~m} / \mathrm{km})$, horizontalité du profil à l'aval du Doubs, reprise de pente caractéristique à partir de Anse $(\mathrm{Pk} 35)$ et importance considérable du champ d'inondation sont les principales caractéristiques morphologiques de la Saône. La combinaison de ces valeurs avec les débits de hautes eaux par le calcul de la vitesse moyenne pour la crue décennale, de la puissance spécifique (figure 2) et de la force tractrice globale, montrent l'existence de fortes discontinuités hydrologiques de l'amont vers l'aval. Cette analyse longitudinale d'une gamme de variables-clés nous permet alors de proposer une sectorisation de l'hydrosystème (figure 3), avec les tronçons traditionnels de la Haute Saône (de Corre à Gray), de la Petite Saône (jusqu'à l'embouchure du Doubs) et de la Grande Saône (jusqu'à Lyon), nos observations montrant toutefois la nécessité de diviser ce dernier, de part et d'autre de Anse, en Saône horizontale, section la plus représentative de l'image de la rivière par la faiblesse des données et l'étendue de la plaine inondable, et en Saône aval, remarquable par la force des variables hydrologiques.

Une analyse de la granulométrie des sédiments fins déposés à proximité du lit mineur et de la densité des matières en suspension au Pk 31 (crues d'hiver 1993-94 et 1994-95) montre que les variations longitudinales de l'énergie de l'hydrosystème ont des effets perceptibles sur le transport de la charge fluviale. La comparaison des images CM (Passega, 1957, Bravard, 1983) de plusieurs sites d'échantillonnage sur la Saône montre en effet des différences dans la capacité de transport de la rivière, en relation avec l'énergie de chaque tronçon (figure 2), et révèle même un déplacement de la granulométrie des sédiments fins de l'amont vers l'aval. De même, la comparaison des concentrations de M.E.S. à l'exutoire de l'immense plaine d'inondation avec d'autres hydrosystèmes (Meybeck, 1985) montre la faiblesse relative des valeurs (maximum de $70 \mathrm{mg} / \mathrm{l}$ ), conséquence de la décantation opérée par les larges et longues plaines d'écrêtement de la Saône. 


\section{III — EFFETS MORPHODY- NAMIQUES DES CRUES SUR LE TRONÇON LE PLUS ACTIF ET LE PLUS ANTHROPISÉ}

D'une remarquable originalité au sein de l'hydrosystème, le tronçon situé entre Anse et Lyon cumule donc les vitesses et les puissances de loin les plus fortes dans un espace où, de surcroît, le cours d'eau et la plaine sont les plus utilisés et aménagés. L'étude diachronique du cours de la Saône aval depuis le milieu du XIXe siècle montre que l'hydrosystème est également affecté, au niveau d'un tronçon de quelques kilomètres, par des changements de la géométrie en plan à une échelle intermédiaire de 10 à 100 ans. Ce type d'étude est particulièrement motivant dans le cas de la Saône, caractérisée par la permanence de son tracé à l'Holocène du fait de l'absence de processus d'évolution naturels énergiques, ce qui nous permet de nous attacher aux seuls effets des aménagements, qu'ils soient directs ou liés à la puissance hydraulique du tronçon. Les transformations morphologiques les plus évidentes se manifestent par la disparition des îles (de 26 en 1845-50 à 11 aujourd'hui) et par la perte de $5,4 \mathrm{~km}$ de linéaire de rives. C'est sur le plan transversal que la métamorphose du lit est la plus remarquable, les aménagements dessinant une nouvelle Saône profonde, encaissée entre des rives hautes et érodées, et se partageant entre le chenal navigable et une étroite plate-forme, relique des platis.

Sous le poids des aménagements et des facteurs hydrauliques, le cours de la Saône aval a donc considérablement changé depuis 150 ans. L'hydrosystème est de plus en plus restreint à son seul chenal d'écoulement qui s'articule autour d'une ripisylve artificialisée dont la complexité et la longueur sont en diminution. La cartographie précise des rives, milieux aujourd'hui déterminants de l'hydrosystème, montre la très forte dominance des berges plus ou moins artificielles, une nette régression de la berge naturelle typique en même temps qu'un "reverdissement" actif des anciens ouvrages. Dans la forme actuelle du cours d'eau, les berges qui offrent la plus grande diversité et les sites les plus attrayants sont alors tous liés à la présence d'une île (figure 4).

\section{IV — DYNAMIQUE D'UN ESPACE RÉSIDUEL : LES ÎLES DE LA SAÔNE AVAL}

Cette observation nous a conduit alors à nous pencher, à l'échelle de deux îles (île des Comtes, île du Roquet, Pk 32), sur les événements plus brefs affectant les milieux riverains en étudiant les effets des crues et des actions humaines sur la ripisylve. L'occupation ancienne des îles, les chablis dus au vent, la gestion des propriétaires et les crues expliquent le paysage actuel des deux îles mais leurs formes d'utilisation discordantes et leur différence de sensibilité aux crues en font des milieux très distincts, tant par la morphologie que par la végétation.

L'approche dendrogéomorphologique (Alestalo, 1971) des arbres de la rive érodée de l'île des Comtes (figure 5), qui permet de retracer l'évolution spatio-temporelle des phénomènes affectant leur milieu de croissance, fait apparaître les années pendant lesquelles les perturbations physiques ont été les plus importantes, à savoir principalement les années 1980. L'érosion des berges de la Saône semble donc être significative depuis une ou deux décennies, sous les effets distincts ou cumulés de l'augmentation de la fréquence des crues, de la fin de la longue période d'années sans crue de 1970 à 1977 , de la baisse du niveau de la Saône d'environ un mètre en 1970, des crues de la trilogie de 1981-83 et des tempêtes de vent. De même, l'analyse des signatures dendrochronologiques de Populus tremula $L$. et Quercus robur $L$. de l'île du Roquet soumis uniquement aux immersions montre les perturbations provoquées par les inondations de printemps. En raison de leur durée, les crues de la Saône 


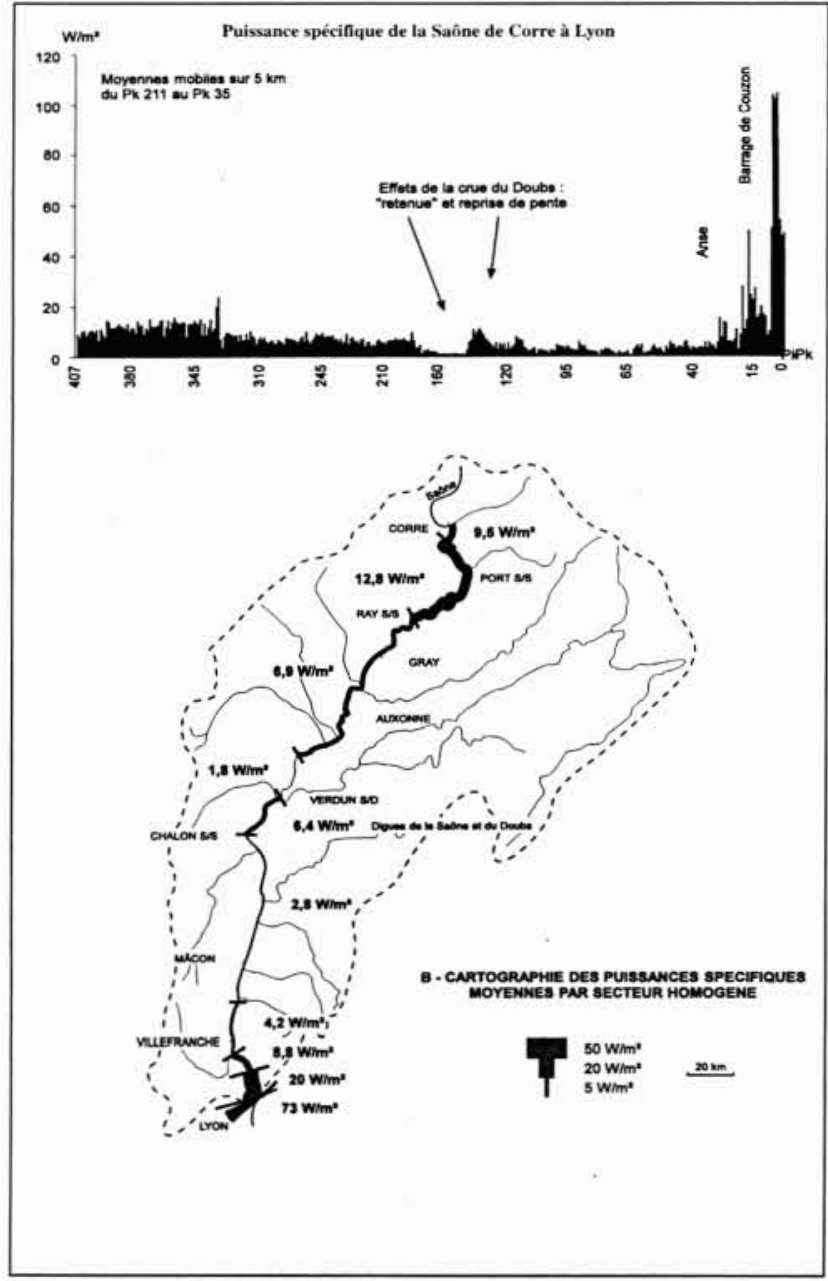

2. Puissance spécifique de la Saône et relation avec la granulométrie des sédiments fins échantillonnés.

semblent avoir un réel impact sur la végétation. Cependant, l'observation des effets des inondations sur la croissance des arbres n'est possible que depuis l'apparition des crues tardives. De même, les arbres de la ripisylve ne réagissent à l'érosion des berges que depuis une à deux décennies. Les perturbations ne se manifestent donc que depuis les dernières années, les nouvelles conditions hydrologiques constituant un paramètre nouveau agissant sur l'équilibre des milieux riverains.

\section{$\mathrm{V} \square$ CONCLUSION}

L'objet de nos recherches et le volume et la qualité des données de base nous ont permis de mettre en œuvre des approches nouvelles dans l'étude des hydrosystèmes fluviaux. Nous avons pu ainsi mettre en évidence l'organisation amont-aval de variables-clés et les disparités longitudinales d'un hydrosystème fluvial. Nous avons pu également quantifier l'évolution morphologique d'un cours d'eau en plusieurs étapes pendant une très longue période. Ce travail participe à l'étude des hydrosystèmes fortement influencés par les activités humaines en s'attachant à un cours d'eau morphodynamiquement stable, voué à de multiples usages depuis des époques très anciennes. Cet ouvrage contribue de plus à la connaissance de la morphologie et de la dynamique des cours d'eau rendus navigables et à la définition des berges

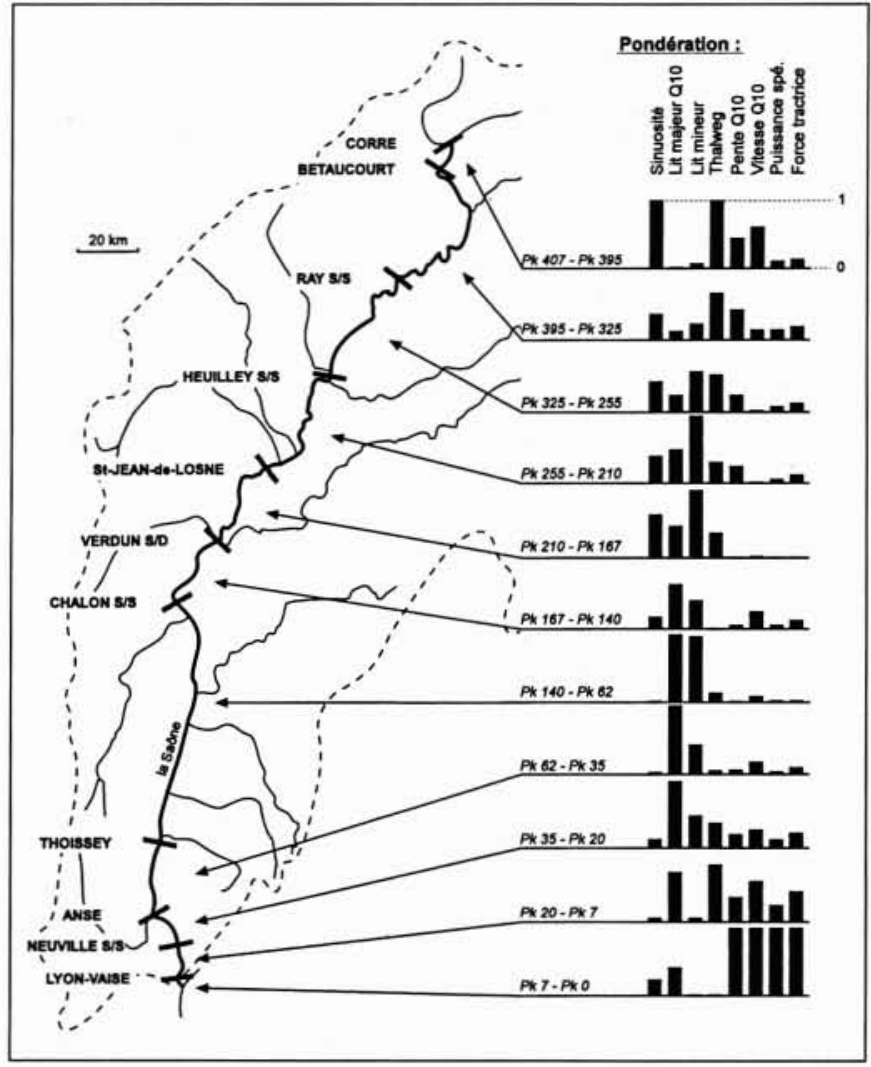

3. Les tronçons homogènes de la Saône de Corre à

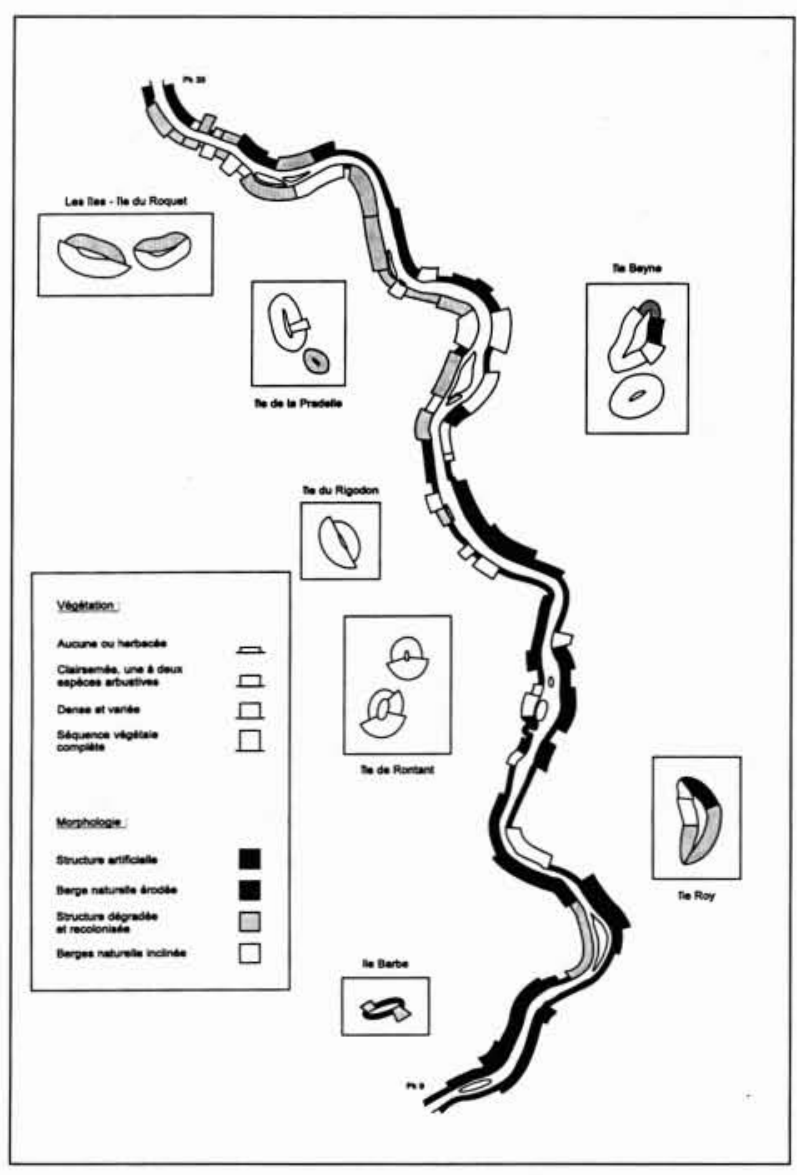

4. Qualité écologique des berges de la Saône aval. 
A - CARTOGRAPHIE DES

FORMES DE CROISSANCE

DES ARBRES

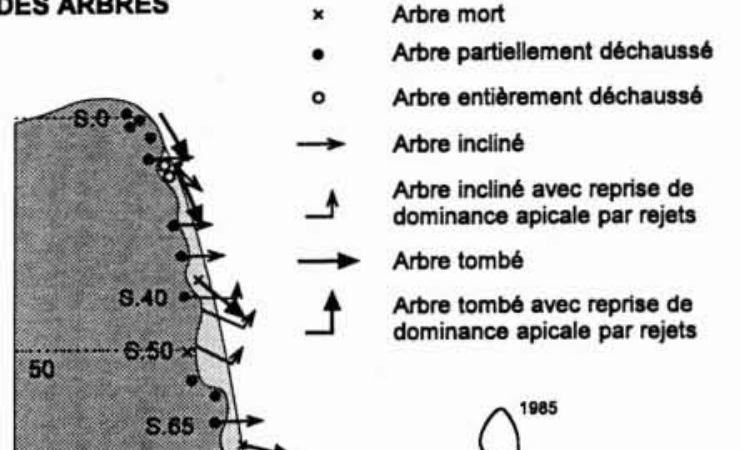

B - ILLUUSTRATION SUR CERTAINS SITES REPRESENTATIFS
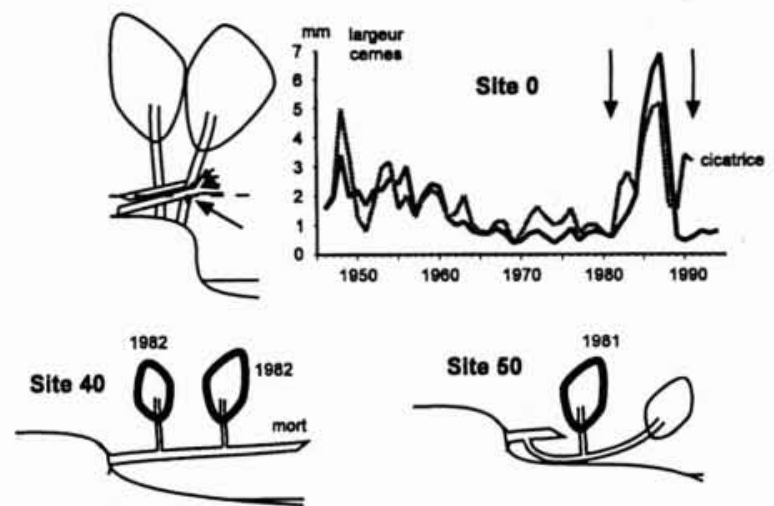

SIte 175
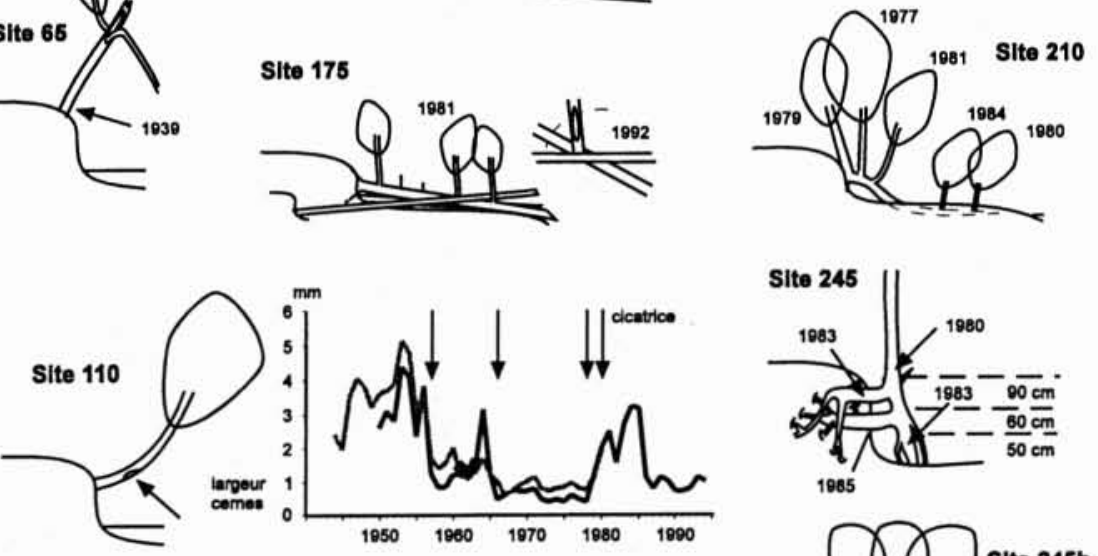

$8.125-125 b$

Site 65
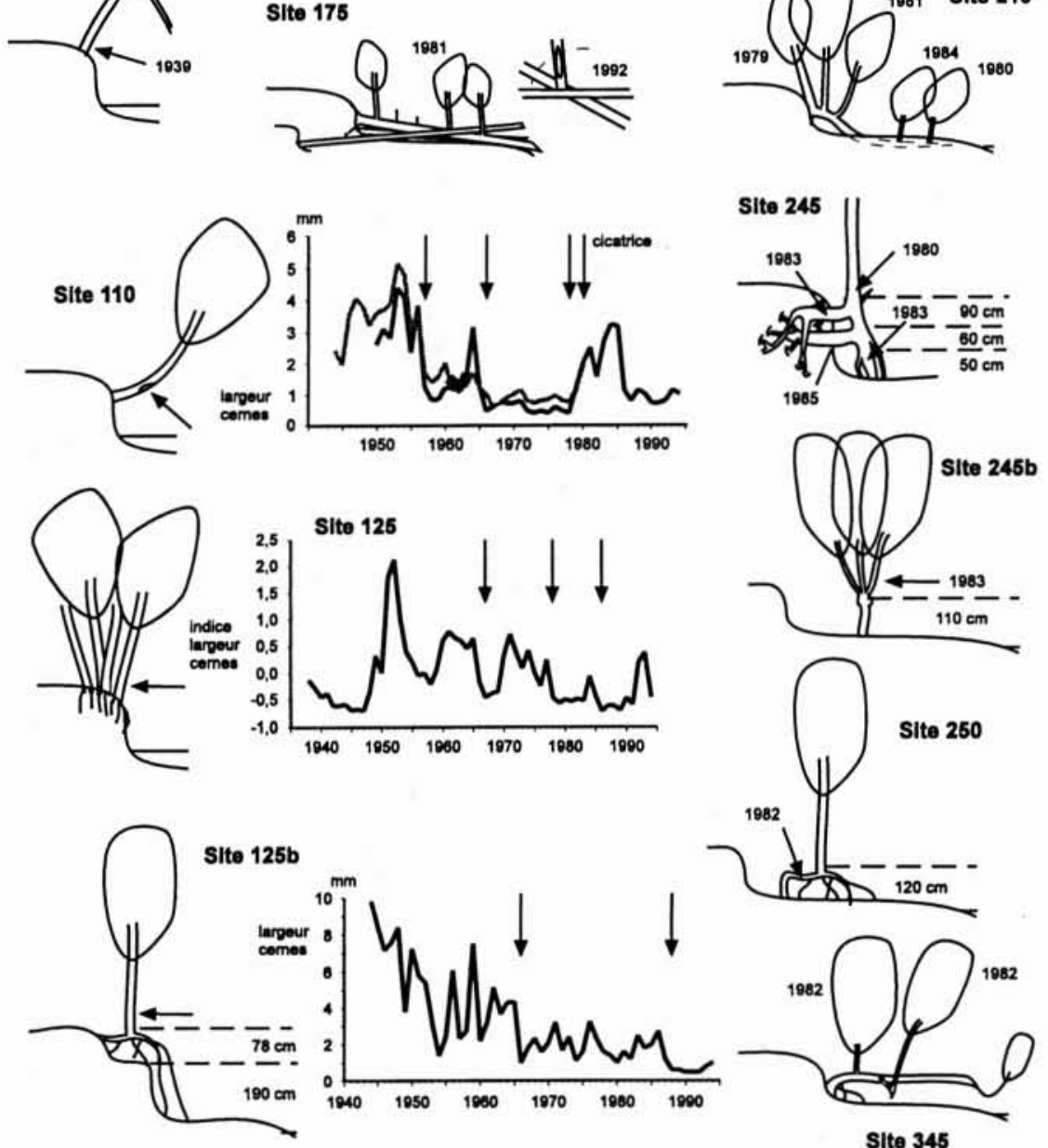

350
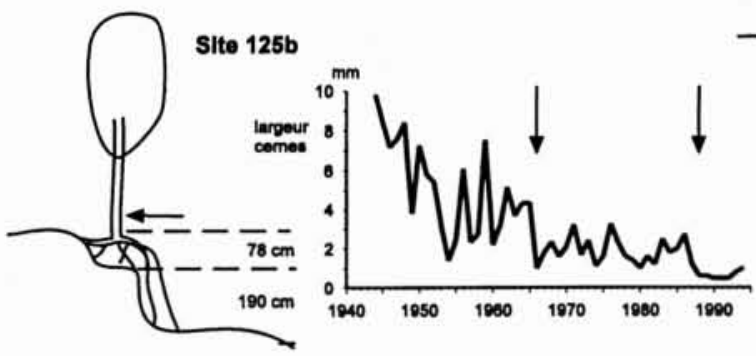

Site 345
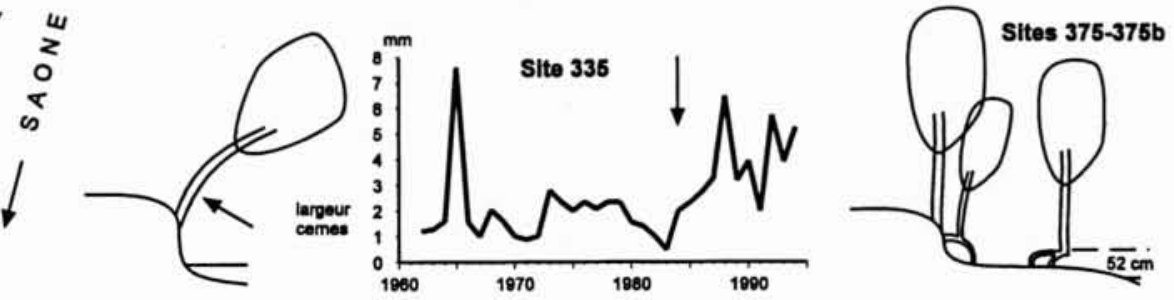

5. Cartographie des formes de croissance des arbres de la berge érodée de l'île des Comtes. 
des tronçons naturels des voies fluviales. Enfin les caractéristiques des crues de la Saône ont également permis la mise en œuvre des méthodes de la dendrogéomorphologie dont nous montrons l'intérêt dans l'étude des hydrosystèmes fluviaux, tout en contribuant aux recherches sur les relations entre les inondations et la croissance des arbres.

\section{Remerciements}

La thèse dont est tiré ce résumé a été présentée devant l'Université Paris IV-Sorbonne le 25 juin 1996. Elle a été réalisée sous la direction de Monsieur J.P. Bravard et en collaboration avec Monsieur Y. Begin (Université Laval, Québec). La diversité des approches a été assurée grâce au Laboratoire de Géographie Physique (Université Lyon III), au Centre d'Etudes Nordiques (Université Laval), au Laboratoire de Botanique Historique \& Palynologie (Université Aix-Marseille) et au laboratoire de géomorphologie Rhodanienne (Université Lyon II). Nous devons enfin l'ensemble des données de base au Service de la Navigation Saône-Rhône (subdivision de Mâcon), à la Compagnie Nationale du Rhône et à l'Agence de Bassin Rhône-Méditerranée-Corse.

\section{Bibliographie sommaire}

[1] Alestalo J., 1971 : Dendrochronological interpretation of geomorphic processes. Fennia, 105, $140 \mathrm{p}$.

[2] Balland P., 1989 : La Saône : problématique des crues "justes débordantes". Actes de la journée d'étude du 17 mars 1989, Rivières en crise, Saône, Ain, Durance, Univ. Lyon III, Inst. de Droit de l'Environnement, p. $17-27$

[3] Bonnamour L., 1981 : La Saône, une rivière, des hommes. Coll. Bennetton, Paris, $125 \mathrm{p}$.

[4] Bravard J.P., (sous presse) : Le profil en long de la Saône et du Rhône dans la région lyonnaise au Tardiglaciaire et à l'Holocène : interférence de la tectonique et de la dynamique fluviale. Géogr. Phys. et Quat., 23 p.

[5] Compagnie Nationale du Rhône - Beture Setame, 1989 : Rivière la Saône : élaboration d'un schéma d'aménagement hydraulique destiné à limiter l'impact des crues justes débordantes. Rapport, 3 phases, 288 p.

[6] Crouslé T., Dubois P., 1972 : L'aménagement de la Saône entre Lyon et Auxonne. Navigation, ports et industries, $n^{\circ} 12$, p. $729-733$

[7] Debelmas J., 1974 : Géologie de la France, les chaînes plissées du cycle alpin et leur avant-pays. Vol. II, éd. Doin, Paris,544 p.
[8] Decamps H., Naiman R.J., 1990 : Towards an ecotone perspective. The ecology and management of aquatic-terrestrial ecotones, Man and the Biosphere series, UNESCO, p. 1-8

[9] Dumorey T., 30 août 1779 : Mémoire au sujet de la navigation sur la Saône par l'ingénieur en chef des Etats de Bourgogne. Archives Nationales, H 157, ler dossier, C-d'Or, C3364, f $172 v^{\circ}$, C2986, 618 p.

[10] Gilvear D.J., Bravard J.P., 1993 : Dynamique fluviale. In Amoros et Petts éd. : Hydrosystèmes fluviaux, coll. écologie, n²4, éd Masson, p. 6182

[11] Journaux A., 1956 : Les plaines de la Saône et leurs bordures montagneuses. Thèse Paris, éd. Caron, Caen, 531 p.

[12] Laval, 1845 : Notice sur les travaux de perfectionnement de la navigation de la Saône, entre l'embouchure du canal du Rhône au Rhin et Lyon. Ann. des Ponts et Chaussées, Mémoires et documents relatifs à l'art des constructions, p. 1-47

[13] Mission déléguée de Bassin Rhône-Méditerrannée-Corse, 1984 : Les crues du bassin de la Saône, rapport technique et projet de conclusions. Rapport d'étude, $158 \mathrm{p}$.

[14] Parde M., 1925 : Le régime du Rhône, étude hydrologique. Inst. des Etudes Rhodaniennes, Lyon, 883 et 440 p.

[15] Rat P. et al., 1984 : Le fossé bressans. Géologie de la France, B.R.G.M., n³, p. 185-19

[16] Shroder J.F., 1978 : Dendrogeomorphology : review and new techniques of tree-ring dating. Progress in Physical Geography, $n^{\circ} 4$ (2), p. 161188

[17] Untermaier J., 1982 : La protection implicite de la nature, le cas du Val de Saône et de la Dombes. La Saône à Trévoux, un site, une rivière, une histoire : pour quel devenir ? Patrimoine des Pays de l'Ain, p. 16-25

[18] Carte de la Saône de Pagny à Lyon au 1/10 000e, "Carte topographique du cours de la Saône flottable et navigable levée et gravée de 1857 à 1866 par les soins de l'Administration des Ponts et Chaussées". Arch. Dép. du Rhône, 1034 W album 7, 23 feuilles.

[19] "Amélioration du cours de la Saône entre Verdun et Lyon par l'établissement de cinq barrages mobiles éclusés : Profil en long général de la Saône entre Corre et Lyon" dréssé par l'Ingénieur en Chef Thiolliere le 26 octobre 1869. Ponts et Chaussées, Service Spécial de la Saône, Musée de Mâcon.

[20] Carte générale du cours de la Saône flottable et navigable au 1/40 000e dressée en 1874, Ponts et Chaussées, Service spécial de la Saône. Archives Départementales de Dijon, série XII S1 à 8, 19 feuilles.

[21] Carte pour la navigation, "La Saône de Corre à Lyon en 1954" (Salagnac).

[22] Carte I.G.N. au 1/25 000e, Lyon 3031 ouest (1988), Villefranche-surSaône 3030 ouest (1984). 